\title{
植物细胞 $\mathbf{H}_{2} \mathbf{O}_{2}$ 的信号转导途径*
}

\section{程艳丽 宋纯鹏**}

(河南大学生命科学学院, 河南省植物逆境生物学重点实验室, 开封 475001)

\begin{abstract}
摘要 光、环境胁迫和植物激素 $\mathrm{ABA}$ 可以引起植物体内 $\mathrm{H}_{2} \mathrm{O}_{2}$ 升高, 而 $\mathrm{H}_{2} \mathrm{O}_{2}$ 作为一个较早进 化出来的信号分子, 不仅在诱导氧化性光合作用中起了关键的作用, 并且可以调节诸如气孔运 动、超敏反应、细胞凋亡和基因表达等许多过程. 细胞内 $\mathrm{H}_{2} \mathrm{O}_{2}$ 浓度必须维持在一种精细平衡状 态, 它一方面可以通过质膜氧化还原系统和光呼吸系统产生, 另一方面也存在完善的清除机制. $\mathrm{H}_{2} \mathrm{O}_{2}$ 从质外体或者产生源进入细胞, 然后进入亚细胞区域. $\mathrm{H}_{2} \mathrm{O}_{2}$ 可以调节信号转导蛋白, 如蛋白 质磷酸化酶、转录因子、以及位于质膜或其它膜上的 $\mathrm{Ca}^{2+}$ 通道. 其中, 蛋白质可逆磷酸化可启动 细胞质和细胞核的下游信号转导, 通过影响转录因子而影响基因的表达; 转录因子通过氧化而激 活自身或诱导其定向转运至细胞核内. 然而, $\mathrm{H}_{2} \mathrm{O}_{2}$ 作为信号分子的研究相对处于“年轻” 阶段, 诸 如细胞如何感受 $\mathrm{H}_{2} \mathrm{O}_{2}$, 以及在细胞感受 $\mathrm{H}_{2} \mathrm{O}_{2}$ 信号转导过程中哪种细胞过程是最主要的或是限速 步骤, 何种基因对 $\mathrm{H}_{2} \mathrm{O}_{2}$ 是特异和必需的等问题仍然所知甚少, 这些问题的破解依赖于功能基因 组学和遗传学分析.
\end{abstract}

\section{关键词 $\mathrm{H}_{2} \mathrm{O}_{2}$ 信号转导 $\mathrm{ABA}$ 环境胁迫 基因表达 细胞调亡}

生命离不开氧, 它对于生命的生存和发展至关 重要. 研究生命如何感受氧化信号, 已成为生命科学 的重大基本课题. 光、环境胁迫(如干旱)和植物激素 $\mathrm{ABA}$ 可以引起植物体内 $\mathrm{H}_{2} \mathrm{O}_{2}$ 升高, 这成为细胞连接 外界环境刺激的重要枢纽之一. 弄清植物对外界因 子的反应机理, 不仅有利于阐明其基本的生物学特 征, 而且对农业生产极为重要. 氧化还原状态的改变 作为一个古老的信号, 存在于细菌到高等动植物的 细胞内 ${ }^{[1]}$. 目前, $\mathrm{H}_{2} \mathrm{O}_{2}$ 作为信号分子作用引起了人们
的极大关注 ${ }^{[2-4]}$.

\section{$1 \mathrm{H}_{2} \mathrm{O}_{2}$ ：生命进化的衍生物或必要的分 子?}

通常认为早期的地球上大气是还原型的(富含 $\left.\mathrm{H}_{2}\right)$ 或者是中性的 $\left(\mathrm{CO}_{2}-\mathrm{N}_{2}\right)$, 相反现在的大气处于高度氧 化状态 $\left(20 \% \mathrm{O}_{2}\right)$. 这些氧的主要来源是光合作用. 然 而, 在生命进化过程中, 在无氧的条件下氧化性的光 合作用如何进化出来, 或者是这种产生氧的能力是 
如何演化的呢? 这是一个非常重要的问题. 大气氧 化剂中非生物性产物可能会提供一种这样的机制: 在空间上限定的栖息地以维持局部的氧化条件, 消 除可用的还原剂, 迫使氧化性的光合生物利用水作 为电子供体. 有人提出大气中的 $\mathrm{H}_{2} \mathrm{O}_{2}$ 在诱导氧化性 光合作用中起了关键的作用, 因为局部环境中过氧 化物的增加, 有机体不但面对还原剂的丧失, 同时还 要被迫发展生化的机制(如过氧化氢酶)以应对氧化条 件的改变. 这些对于保护生命体免遭氧化性光合作 用产物伤害是必须的 ${ }^{[5]}$. 因此在无氧环境中, 这种条 件可以迫使地球让的早期氧化性光合作用演化出来.

\section{$2 \mathrm{H}_{2} \mathrm{O}_{2}$ 平衡(homoestasis): 产生和清除}

在正常的代谢条件下, 细胞可以从不同的渠道 产生 $\mathrm{H}_{2} \mathrm{O}_{2}$. 细胞内 $\mathrm{H}_{2} \mathrm{O}_{2}$ 浓度变化范围较大, 如拟南 芥中 $0.06 \sim 7 \mathrm{mmol} / \mathrm{L}^{[6]}$, 而玉米和水稻中为 $1 \sim 2$ $\mathrm{mmol} / \mathrm{L}^{[7]}$, 然而, 人们至今仍不知道不同亚细胞区域 的 $\mathrm{H}_{2} \mathrm{O}_{2}$ 含量的差异. 尽管对细胞毒害的精确胞间 $\mathrm{H}_{2} \mathrm{O}_{2}$ 浓度不同, 但是细胞通常通过非常有效的抗氧 化剂系统平衡高浓度 $\mathrm{H}_{2} \mathrm{O}_{2} . \mathrm{H}_{2} \mathrm{O}_{2}$ 存在的寿命较长, 具 有较高的跨膜通透性并能在植物细胞间迅速扩散; 同时, 外界刺激能迅速的刺激其合成和分解等特 点 ${ }^{[8,9]}$, 符合胞间信号所有的重要标准, 因而成为人 们比较关注的氧化信号分子. 然而, $\mathrm{H}_{2} \mathrm{O}_{2}$ 在何种条件 下分别作为信号分子和对细胞产生毒性, 仍然是一 个悬而未决的问题. 通常, 起信号作用 $\mathrm{H}_{2} \mathrm{O}_{2}$ 在细胞内 的浓度为 $1 \sim 700 \mathrm{nmol} / \mathrm{L}$ 之间 ${ }^{[10]}$.

\section{$2.1 \mathrm{H}_{2} \mathrm{O}_{2}$ 的产生}

在植物细胞正常代谢过程中, 活性氧(reactive oxygen species, ROS)可由多种途径产生. 质膜上的氧 化还原酶系统(plasma membrane redox system, PMRS) 是产生 $\mathrm{H}_{2} \mathrm{O}_{2}$ 的重要部位 ${ }^{[11]}$, 同时植物细胞壁中的过 氧化物酶和叶绿体PSI也可能与植物氧化猝发有关.

已知产生 $\mathrm{H}_{2} \mathrm{O}_{2}$ 的NADPH氧化酶是PMRS的一个 重要成分. 植物拟南芥基因组存在 10 个NADPH氧化 酶的同源基因, 但是直到最近才证明这些基因在产 生 $\mathrm{H}_{2} \mathrm{O}_{2}$ 中的作用和功能 ${ }^{[12 \sim 14]}$. 植物中氧化猝发 (oxidative burst, OXB) 和哺乳动物细胞激活嗜中性细
胞相似, 是由NADPH氧化酶的激活而产生的 ${ }^{[15]}$. 用 In-gel激酶分析，在土豆和烟草细胞质膜鉴定到产生 $\mathrm{O}_{2}^{-\cdot}$ (因而产生 $\mathrm{H}_{2} \mathrm{O}_{2}$ ) 的活性 ${ }^{[16]}$, 这种活性直接被 $\mathrm{Ca}^{2+}$ 激活. 用DPI作为抑制剂也在保卫细胞质膜鉴定到了 NADPH参与 ABA诱导 $\mathrm{H}_{2} \mathrm{O}_{2}$ 的产生 ${ }^{[17,18]}$. 用拟南芥 AtrobhD和AtrobohF基因的敲除突变体证实 $\mathrm{H}_{2} \mathrm{O}_{2}$ 产生 需要NADPH氧化酶 ${ }^{[12]}$. 用病毒诱导基因沉默(VIGS) 方法, 发现细胞积累 $\mathrm{H}_{2} \mathrm{O}_{2}$ 需要 NbrbohA 和 $N$ brbroh $B^{[14]}$. 位于质膜外侧的过氧化物酶、 NADPH 氧化酶及多胺氧化酶等都可以产生 $\mathrm{H}_{2} \mathrm{O}_{2}$, 质膜上还 原型的电子载体也可以氧化产生 $\mathrm{H}_{2} \mathrm{O}_{2}$ 和 $\mathrm{O}_{2}^{-*}$, 后者经 歧化作用可形成 $\mathrm{H}_{2} \mathrm{O}_{2}$.

除质膜NADPH氧化酶外, $\mathrm{H}_{2} \mathrm{O}_{2}$ 也可由一种 $\mathrm{pH}$ 依 赖性的细胞壁过氧化物酶(POD)产生 ${ }^{[19]}$. 病原菌侵染 植物细胞时, 在该酶的催化下, $\mathrm{O}_{2}$ 迅速被还原为 $\mathrm{O}_{2}^{-}$和 $\mathrm{H}_{2} \mathrm{O}_{2}{ }^{[20]}$. 质膜上NADPH氧化酶可与胞壁POD起串联 作用. 同时, 光合电子传递系统是植物细胞中 ROS的 一个重要来源, 叶绿体光合电子传递链PS I 的受体 端存在大量的自动氧化酶类, 能够通过米勒反应将 氧还原成超氧化物, 超氧阴离子可通过酶促反应歧 化成 $\mathrm{H}_{2} \mathrm{O}_{2}$ 和 $\mathrm{O}_{2}$. PS II 的抑制剂atrazine也可强烈抑制 强光条件下气孔保卫细胞中 $\mathrm{H}_{2} \mathrm{O}_{2}$ 的产生 ${ }^{[8]}$.

\section{$2.2 \mathrm{H}_{2} \mathrm{O}_{2}$ 的清除}

细胞内 $\mathrm{H}_{2} \mathrm{O}_{2}$ 浓度必须处在一种精细的平衡状态, 因此在进化过程中植物体已形成了复杂和有效的氧 化应激机制. 植物体内有效清除 $\mathrm{H}_{2} \mathrm{O}_{2}$ 的机制分为酶 促和非酶促两类: 酶促类包括过氧化氢酶 $(\mathrm{CAT})$ 、抗 坏血酸过氧化物酶 (APX) 和谷胱甘肽过氧化物酶 (GPX) 等 ${ }^{[21]}$; 非酶促类包括抗坏血酸、谷胱甘肽、甘 露醇和类黄酮等.

CAT可以催化 $\mathrm{H}_{2} \mathrm{O}_{2}$ 生成 $\mathrm{H}_{2} \mathrm{O}$ 和氧, 它是第一个纯 化和结晶的抗氧化酶. 植物中CAT可以清除线粒体电 子传递、脂肪酸 $\beta$-氧化和最为重要的呼吸氧化产生的 $\mathrm{H}_{2} \mathrm{O}_{2}$. 在拟南芥基因组中存在 3 种编码不同过氧化氢 酶的基因Cat1, Cat2 和Cat3. 生物和非生物胁迫可以 促使 $\mathrm{Ca}^{2+}$ 流入细胞, 胞内 $\mathrm{Ca}^{2+}$ 的增加可以刺激 $\mathrm{H}_{2} \mathrm{O}_{2}$ 产 生, 它可以作为信号分子, 引起相应的生理反应 ${ }^{[22]}$. 
胞内 $\mathrm{Ca}^{2+} / \mathrm{CaM}$ 水平的提高可以刺激 CAT活性, 降低 胞内 $\mathrm{H}_{2} \mathrm{O}_{2}$ 的水平 ${ }^{[23]}$. CAT的缺失可以很明显地影响强 光引起的基因表达的变化 ${ }^{[24]}$.

APX需要抗坏血酸和GSH再生系统, 即抗坏血 酸-谷胱甘肽循环. 在叶绿体基质或类囊体膜上, ASA-POD可将 $\mathrm{H}_{2} \mathrm{O}_{2}$ 分解为 $\mathrm{H}_{2} \mathrm{O}$. 除 $\mathrm{O}_{2}$ 外, 单脱氢抗 坏血酸 $(\mathrm{MDA})$ 是光合电子另一个受体分子, 其在体 内的产生和减少直接影响Mehler反应中 $\mathrm{H}_{2} \mathrm{O}_{2}$ 的调节. 和其他生物不同, 植物有多个基因编码 SOD和 APX, 分别分布在叶绿体、线粒体、过氧化物酶体、细胞质 和质外体. 当保卫细胞中抗坏血酸(ASC)氧化还原状 态升高, 可以降低其对 $\mathrm{H}_{2} \mathrm{O}_{2}$ 反应和 $\mathrm{ABA}$ 信号转导 ${ }^{[25]}$. 拟南芥缺失细胞质中APX1, 叶绿体内整个 $\mathrm{H}_{2} \mathrm{O}_{2}$ 清除 系统的功能都会丧失 ${ }^{[26]}$. 当保卫细胞具有较高ASC 的水平时, 气孔对 $\mathrm{H}_{2} \mathrm{O}_{2}$ 和ABA的信号不敏感 [27].

在拟南芥基因组中 $G P X$ 家族含有 7 个基因, 其中 几个基因可被生物胁迫所诱导 ${ }^{[28]}$. 令人兴奋的是, 在 酵母中发现一个谷胱甘肽过氧化物酶 (Gpx3) 可以作 为 $\mathrm{H}_{2} \mathrm{O}_{2}$ 的受体和氧化还原信号传递子, 激活基因的 表达 ${ }^{[29]}$. 同时, 酵母质膜 SLN1(synthetic lethal of $\mathrm{N}$-end rule1)组氨酸激酶高度对 $\mathrm{H}_{2} \mathrm{O}_{2}$ 敏感, 可以作为 过氧化的感应子 ${ }^{[30]}$. 拟南芥基因组含有几个SLN1 同 源体, 有可能一个或较多编码这些蛋白的基因作为 过氧化的感应子起作用.

作为光呼吸途径的组成部分, $\mathrm{H}_{2} \mathrm{O}_{2}$ 在过氧化物 酶体中形成. 但是, 植物在进化过程中也相应地产生 了光氧化保护机制, 其主要目的是调节光合作用. 在 上述条件下, 为阻止电子传递链的过度还原, 植物演 化出光呼吸途径产生 NADP ${ }^{+[31]}$. 另一方面, 通过光 呼吸在过氧化物酶体产生的 $\mathrm{H}_{2} \mathrm{O}_{2}$ 可以作为信号分子, 并可以影响叶片抗氧化系统的氧化还原状态, 尤其 是在干旱条件下更是如此 ${ }^{[32]}$.

\section{$3 \quad \mathrm{H}_{2} \mathrm{O}_{2}$ 信号转导途径}

\section{1 刺激与感受}

(1) 激发子和氧化老发: 人们普遍认为植物具有 一种先天的“免疫系统”一一氧化猝发, 即植物细胞在 胁迫条件下能迅速产生一些活性氧分子, 进而启动
体内其他信号级联过程, 引起一些特有的生理反应.

在植物-病原菌互作过程中, ROS的产生通常十 分迅速, 其对应的OXB模式也有所不同. Baker等 人 ${ }^{[33]}$ 和Levine等人 ${ }^{[34]}$ 用非亲和性病原菌分别处理烟 草和大豆细胞, 发现胞内 $\mathrm{H}_{2} \mathrm{O}_{2}$ 的产生具有双时相特 征. 时相 I (1 2 h) 是一个快速和短暂的过程, 此过程 可被一些亲和性及非亲和性病原菌诱导产生; 而时 相 II (2 5 h) 出现较为缓慢且持续时间较长, 此过程 仅为非亲和性病原菌与植物互作 (不相容系统)所特有. 在非亲和性病原菌与植物互作过程中, 时相 II 与质 膜 $\mathrm{H}^{+}$的吸收 $/ \mathrm{K}^{+}$外流相偶联. 现已证实, 时相 II 是抗 病基因与无毒基因相互作用的后续反应，与植物的 抗病性密切相关 ${ }^{[15]}$.

(2) 水杨酸: 水杨酸 $(\mathrm{SA})$ 作为植物抗病信号分子 目前正引起人们更多的注意, 尤其在OXB及系统获 得性抗性(SAR)形成过程中, SA起着重要作用. 已知 $\mathrm{OXB}$ 产生的 $\mathrm{H}_{2} \mathrm{O}_{2}$ 和SA可能是诱导SAR信号转导途径 的中间成分 ${ }^{[7]}$, 但二者在抗病信号转导过程中的地位 还有待研究. SA可通过抑制细胞内的CAT而促进胞内 $\mathrm{H}_{2} \mathrm{O}_{2}$ 的累积 ${ }^{[35]}$, 还可作用细胞质膜NADPH氧化酶促 进 $\mathrm{H}_{2} \mathrm{O}_{2}$ 的产生 ${ }^{[36]}$; 同时, 较高浓度的 $\mathrm{H}_{2} \mathrm{O}_{2}$ 也可诱导 $\mathrm{SA}$ 的合成. 总之, $\mathrm{OXB}$ 过程中, $\mathrm{SA}$ 可在 $\mathrm{H}_{2} \mathrm{O}_{2}$ 的上游或 下游起作用. 同时, $\mathrm{SA}$ 可以诱导保卫细胞 $\mathrm{H}_{2} \mathrm{O}_{2}$ 的产 生，并导致气孔的关闭 ${ }^{[37]}$.

(3) ABA：水分胁迫下植物激素ABA在细胞中积 累并诱导一系列胁迫适应反应. 在拟南芥中 $\mathrm{ABA}$ 可 以刺激 $\mathrm{H}_{2} \mathrm{O}_{2}$ 积累, 并通过激活 $\mathrm{Ca}^{2+}$ 通道活性诱导气 孔的关闭 ${ }^{[13,38]}$. 与此同时, 苗雨晨等人 ${ }^{[17]}$ 和张骁等 人 ${ }^{[18]}$ 发现ABA在诱导虫豆气孔关闭过程中有 $\mathrm{H}_{2} \mathrm{O}_{2}$ 的 产生, 其产生的位点可能在质膜NADPH氧化酶和叶 绿体, 因此产生的 $\mathrm{H}_{2} \mathrm{O}_{2}$ 可能是ABA诱导气孔关闭过 程中信号转导链的一个中间成分.

\section{2 信号转换}

(1) MAPK：促分裂原活化蛋白激酶 (mitogenactivated protein kinase, MAPK)以蛋白磷酸化的形式 把细胞外信号刺激传递到细胞内, 同时特异性的放 大信号. 这个信号转导途径的 3 个核心组成元件依次 为: MAPKKK, MAPKK (MEK) 和MAPK ${ }^{[39]}$. MAPK介 
导的级联反应参与很多种胁迫和激素信号转导过程. 用外源的 $\mathrm{H}_{2} \mathrm{O}_{2}$ 处理拟南芥叶肉原生质体，激活了与 烟草细胞NPK相似的拟南芥ANP激酶, 然后激活 MAPK的上游因子, 启动了 MAPK级联的信号传递, 最终导致谷胱甘肽-S-转移酶(GST)基因的表达 ${ }^{[40]}$. $\mathrm{H}_{2} \mathrm{O}_{2}$ 也能增加拟南芥核甘酸二磷酸 (NDP) 激酶 2 的表 达 ${ }^{[41]}$. 超表达 $N D P K 2$ 可以减少 $\mathrm{H}_{2} \mathrm{O}_{2}$ 的积累, 提高包 括冷、氧化和盐等多种胁迫的抗性. 这些数据表明, 不同的胁迫可以诱导ROS的产生, 然后激活MAPK信 号转导链. 用MEK $1 / 2$ 的特异性抑制剂(PD98059)处 理蚕豆叶片的表皮, 研究了MEK $1 / 2$ 在ABA和 $\mathrm{H}_{2} \mathrm{O}_{2}$ 诱 导气孔关闭信号转导过程中的作用, 发现MAPK在 $\mathrm{H}_{2} \mathrm{O}_{2}$ 介导的 $A B A$ 引起气孔关闭信号转导链上有着双 重的作用: 一是通过激活 $\mathrm{H}_{2} \mathrm{O}_{2}$ 产生系统和抑制清除 系统的酶活性迅速放大氧化信号, 促进 $\mathrm{ABA}$ 诱导的 虫豆气孔关闭; 二是MAPK可以调节 $\mathrm{H}_{2} \mathrm{O}_{2}$ 信号反应 的专一性 ${ }^{[42]}$. 但是, 至今仍未在保卫细胞中克隆出特 异MEK $1 / 2$ 基因, 以显示其控制气孔的反应.

(2) G-蛋白: 许多工作已经证明 Rop(Rho-like small $\mathrm{G}$ protein)可以调节 $\mathrm{H}_{2} \mathrm{O}_{2}$ 的产生 ${ }^{[43]}$. 通过对拟南 芥Rop信号转导的突变体分析, 提出了其作用的模型: Rop可调节乙醇脱氢酶(ADH)基因的表达和植物对缺 氧的忍耐. 低氧激活Rop信号转导, RopGTP酶激活 $\mathrm{H}_{2} \mathrm{O}_{2}$ 产生, 可能是通过NADPH氧化酶, 而后 $\mathrm{H}_{2} \mathrm{O}_{2}$ 诱 导ADH和Rop失活蛋白RopGAP4 基因的表达. 这种 通过RopGAP4 的负调节作用是控制 $\mathrm{H}_{2} \mathrm{O}_{2}$ 产生所必需 的 ${ }^{[44]}$, 从而强调了 Rops在调节所有 $\mathrm{H}_{2} \mathrm{O}_{2}$ 产生过程中 起重要作用, 这种证据令人兴奋. 依赖 $\mathrm{Rop}$ 的 $\mathrm{H}_{2} \mathrm{O}_{2}$ 诱 导可被DPI抑制, 因此这强有力地说明在激活质膜有 关 $\mathrm{NADPH}$ 氧化酶的过程中植物 Rop的功能和人类Rac 相同. 现以证明植物细胞中也存在 $\mathrm{gp} 91^{\text {phox }}$, 但是不

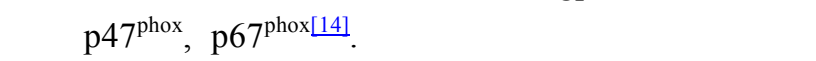
在另外新的植物蛋白, 可以作为其调节分子, 但是这 需要新的研究证据说明植物是否通过其它的机制调 节 $\mathrm{H}_{2} \mathrm{O}_{2}$ 的产生.

(3) $\mathrm{NO}: \mathrm{NO}$ 和 $\mathrm{H}_{2} \mathrm{O}_{2}$ 协同作用参与植物应答生物 胁迫和非生物胁迫的反应 ${ }^{[45,46]}$, 如: NO和ROS相互作 用调节根源逆境信号诱导的 $\mathrm{BBA}$ 合成 ${ }^{[46]}, \mathrm{NO}$ 可增强 植物的耐旱性 ${ }^{[47]}$, 清除强光诱导的ROS, 以减轻植物
的光氧化胁迫伤害 ${ }^{[48}$ 等. 目前, 许多报道表明NO作 为信号分子参与保卫细胞 $\mathrm{ABA}$ 信号转导过程 ${ }^{[49-53]}$, 其调节气孔运动的机制可能是通过激活质膜 $\mathrm{Cl}^{-}$通 道、抑制质膜内向 $\mathrm{K}^{+}$通道 ${ }^{[511}$ 以及调节保卫细胞 $\mathrm{MAPK}$ 的活性等 ${ }^{[54.55]}$.

在植物中, $\mathrm{NO}$ 和 $\mathrm{H}_{2} \mathrm{O}_{2}$ 的相互作用受到较多的关 注 ${ }^{[56.57]}$. 在葛豆悬浮细胞中, 诱导细胞超敏感死亡需 要 $\mathrm{NO}$ 和 $\mathrm{H}_{2} \mathrm{O}_{2}$ 的浓度的平衡 ${ }^{[56]}$. Lum等人 ${ }^{[58]}$ 发现 $\mathrm{H}_{2} \mathrm{O}_{2}$ 作为上游信号诱导 $\mathrm{NO}$ 的产生, 同时, 钙离子介导了 $\mathrm{ABA}$ 诱导的NO产生, 因为它可以被钙离子通道阻断 剂verapamil所抑制. 另外, 有结果显示在ABA信号转 导途径中 $\mathrm{NO}$ 很可能作为 $\mathrm{H}_{2} \mathrm{O}_{2}$ 的上游信号起作用, 并 且磷酸酶和 $\mathrm{Ca}^{2+}$ 也参与其调节 ${ }^{[59]}$. 尽管已经有人把 $\mathrm{ABA}, \mathrm{NO}$ 和 $\mathrm{H}_{2} \mathrm{O}_{2}$ 联系起来, 但有关ABA信号转导过 程中三者之间的相互关系的研究还很缺乏.

(4) 细胞质中 $\mathrm{Ca}^{2+}$ : 已有的实验表明, 用激发子 处理植物细胞, 可导致胞外 $\mathrm{Ca}^{2+}$ 内流. 在悬浮培养的 植物细胞体系中, 加入 $\mathrm{Ca}^{2+}$ 鳌合剂EGTA, 可有效地 抑制细胞的过敏死亡和植物抗毒素(PA)的合成; 并且 在激发子诱导的植物细胞防御反应中, 胞外 $\mathrm{Ca}^{2+}$ 及其 内流可能是信号转导中的最初反应 ${ }^{[9]}$. Miura等 人 [60]报道, 在马铃薯块茎组织切片或悬浮培养细胞, 同 时加入激发子和外源 $\mathrm{Ca}^{2+}$ 鳌合剂, 可有效地抑制OXB. 这表明其作用位点在细胞死亡和PA形成之前, 当有 外源 $\mathrm{Ca}^{2+}$ 存在时, 加入 $\mathrm{Ca}^{2+}$ 载体 $\mathrm{A}_{23187}$ 可有效地激发 $\mathrm{OXB}$ ，而无 $\mathrm{Ca}^{2+}$ 时，则无此作用 ${ }^{[60]}$. 同样，当用 cryptogein处理烟草细胞时, $\mathrm{Ca}^{2+}$ 内流出现在氧化猝发 之前, 并持续 $60 \mathrm{~min}$ 之久 ${ }^{[61]} ; \mathrm{H}_{2} \mathrm{O}_{2}$ 形成之后, 仍有较 高的 $\mathrm{Ca}^{2+}$ 内流 ${ }^{[34]}$. 若用非亲和性病原菌或 $\mathrm{H}_{2} \mathrm{O}_{2}$ 处理 大豆细胞, 则胞外 $\mathrm{Ca}^{2+}$ 内流对诱导细胞的死亡是十分 必要的. $\mathrm{Ca}^{2+}$ 通道阻断剂 $\left(\mathrm{La}^{3+}\right)$ 可阻止细胞的诱导死 亡, 而 $A_{23187}$ 则可促进细胞的死亡. 豇豆受到锈病真 菌侵染时, 细胞质中 $\left[\mathrm{Ca}^{2+}\right]$ 升高, 因此认为这是细胞 过敏反应的重要信号 ${ }^{[62]}$. 由此说明, 细胞质中 $\mathrm{Ca}^{2+}$ 信 号在OXB的上游和下游均起着重要作用.

\section{3 反应}


(1) $\mathrm{H}_{2} \mathrm{O}_{2}$ 与气孔运动: 许多环境胁迫 (如干旱、盐 碱等)可导致保卫细胞内 $\mathrm{H}_{2} \mathrm{O}_{2}$ 的积累, 进而促进气孔 关闭. McAinsh等人 ${ }^{[63]}$ 观察到 $\mathrm{H}_{2} \mathrm{O}_{2}$ 和 $\mathrm{O}_{2}^{--}$可增加保卫 细胞中游离 $\mathrm{Ca}^{2+}$ 的浓度, 通过 $\mathrm{Ca}^{2+}$ 信号系统调节气孔 的运动. 用膜片钳全细胞记录技术研究发现, $\mathrm{H}_{2} \mathrm{O}_{2}$ 能 促进气孔关闭是因为它抑制了保卫细胞质膜内向 $\mathrm{K}^{+}$ 通道, 而 $\mathrm{K}^{+}$的流出和溶质丧失都会引起膨压降低, 促进气孔关闭 ${ }^{[64,65]}$. 同时, $\mathrm{H}_{2} \mathrm{O}_{2}$ 可影响气孔保卫细胞 $\mathrm{pH}^{[66]}$.

更多的研究表明, $\mathrm{H}_{2} \mathrm{O}_{2}$ 作为信号分子调节了气 孔保卫细胞的运动，其调节过程与 $\mathrm{ABA}$ 对气孔运动 的调节有许多类似之处. 已知, $\mathrm{H}_{2} \mathrm{O}_{2}$ 可能作为正调节 因子参与了 $\mathrm{ABA}$ 对气孔运动的调节 ${ }^{[17,38]}$. ABA通过 $\mathrm{NADPH}$ 氧化酶激活保卫细胞内 $\mathrm{H}_{2} \mathrm{O}_{2}$ 的合成, $\mathrm{H}_{2} \mathrm{O}_{2}$ 可 以极低的浓度 $(\mathrm{mmol} / \mathrm{L}$ 级 $)$ 参与 $\mathrm{ABA}$ 诱导的气孔关闭 和激活质膜钙离子通道 ${ }^{[33]}$. Schroeder等人 ${ }^{[67]}$ 提出, $\mathrm{H}_{2} \mathrm{O}_{2}$ 可能作为 $\mathrm{ABA}$ 途径与非 $A B A$ (non-ABA)途径的 胁迫信号与保卫细胞氧化还原状态对气孔运动调节 的交叉位点, 如病原菌感染植物引起保卫细胞中产 生ROS, 导致气孔关闭. 最近发现, ABA诱导保卫细 胞内 $\mathrm{H}_{2} \mathrm{O}_{2}$ 产生和积累的过程中, 磷脂酰肌醇 3-磷酸 (phosphatidylinositol 3-phosphate, PI3P)正调节了 $\mathrm{H}_{2} \mathrm{O}_{2}$ 的产生 ${ }^{[68]}$.

对ABA不敏感突变体abi1-1 和abi2-1 遗传学分析 表明, 蛋白磷酸化也参与了保卫细胞的信号转导过 程. ABI1 和 $\mathrm{ABI} 2$ 编码蛋白质磷酸化酶 $2 \mathrm{C}$ 类的酶, 二 者都参与了保卫细胞关闭过程. 已经证明, 在 $a b i 1$ 中 ABA不能诱导 $\mathrm{H}_{2} \mathrm{O}_{2}$ 的产生, 相反, $a b i 2$ 突变体中仍能 产生 $\mathrm{H}_{2} \mathrm{O}_{2}{ }^{[59]}$, 说明ABI1 可能在 $\mathrm{H}_{2} \mathrm{O}_{2}$ 信号转导的上游, 而 $\mathrm{ABI} 2$ 则在其下游.

ost 1 突变体对ABA不敏感. ABA可以激活野生型 保卫细胞原生质体OST1 激酶活性, 但是ost1 突变体 中却相反: ost1 中 ABA不能诱导 $\mathrm{H}_{2} \mathrm{O}_{2}$ 产生, 但是 $\mathrm{H}_{2} \mathrm{O}_{2}$ 仍能诱导气孔的关闭 ${ }^{[69]}$. 有可能OST1 调节的 $\mathrm{H}_{2} \mathrm{O}_{2}$ 直 接作用于NADPH氧化酶. 尽管这种理论非常有吸引 力, 但是仍缺乏实验证据.

另外, 气孔在下午关闭, 而保卫细胞内 $\mathrm{H}_{2} \mathrm{O}_{2}$ 产 生在早上较低, 上午开始升高, 下午 2 点左右达到高
峰, 然后下降. 这种日变化的模式和保卫细胞的ASC 氧化还原状态有关 ${ }^{[25]}$, 因此脱氢抗坏血酸还原酶 (DHAR)作用是保持ASC循环的基本水平, 这样就不 足清除下午高水平的 $\mathrm{H}_{2} \mathrm{O}_{2}$ 导致气孔的关闭，从而抑 制DHAR表达可以增加干旱的抗性.

(2) $\mathrm{H}_{2} \mathrm{O}_{2}$ 与细胞调亡: 与植物超敏反应 (hypersensitive response, HR)有关的细胞死亡是一种 程序性死亡. 植物受到病原菌侵染时, 可产生氧化猝 发, 在极短的时间内积累活性氧 ${ }^{[19,70,71]}$. 由HR引起的 膜脂过氧化过程中, 某种信号物质在植物体内传递, 这种信号物质可能是不饱和脂肪酸过氧化的代谢产 物. $\mathrm{H}_{2} \mathrm{O}_{2}$ 是细胞凋亡中的主要因子, 但是 $\mathrm{H}_{2} \mathrm{O}_{2}$ 引起的 细胞死亡的分子机制仍然不清楚. 几个方面的研究 证明 $\mathrm{H}_{2} \mathrm{O}_{2}$ 可能作用于线粒体，如用 $\mathrm{H}_{2} \mathrm{O}_{2}$ 处理拟南芥 的细胞, 可以引起线粒体中 $\mathrm{H}_{2} \mathrm{O}_{2}$ 的升高, 导致其功能 的改变和细胞凋亡 ${ }^{[711}$. ROS可直接作为信号传递过程 中的中间体, 在转录水平上激活和调控植物体内各 种防御相关基因的表达. 同时, ROS还可能与NO相互 作用激活过敏细胞的死亡 ${ }^{[50]}$. 转基因拟南芥中组成 型激活AtMEK4 和AtMEK5, 可以打破氧化还原的平 衡, 产生 $\mathrm{H}_{2} \mathrm{O}_{2}$, 诱导细胞死亡 ${ }^{[72]}$.

(3) $\mathrm{H}_{2} \mathrm{O}_{2}$ 与基因的表达: 在高等动物细胞中, $\mathrm{H}_{2} \mathrm{O}_{2}$ 是一个多效活化因子, 作为胞内第二信使它可 激活转录因子的原始类型核因子 $\kappa \mathrm{B}(\mathrm{NF}-\mathrm{\kappa B})$, 并对各 种不同刺激作出迅速的反应 ${ }^{[73]}$. 在拟南芥中发现 NIM1 基因所编码的蛋白与NF- $\kappa$ B的抑制性亚单位 I $\kappa$ B 有许多相似之处. 据报道, NIM1 基因是在植物细 胞防御反应的局部基因表达和SAR的形成过程中起 作用 ${ }^{[74]}$. 另外, $\mathrm{H}_{2} \mathrm{O}_{2}$ 也可作为胞内信使激活转录因子 和促进细胞外信号对基因表达的调控. 植物细胞中 也存在Ref- 1 的类似物. 在动物细胞中, Ref- 1 是一个 氧化还原因子, 当其受到氧化胁迫时, 即可调节 Fos-Jun二聚体与AP-1 顺式作用元件结合, 于是氧化 胁迫应答基因即被活化和得以表达 ${ }^{[75]}$.

$\mathrm{H}_{2} \mathrm{O}_{2}$ 调节很多基因的表达, 包括那些编码抗氧 化剂的酶和 $\mathrm{H}_{2} \mathrm{O}_{2}$ 产生的调节因子. 因此, 细胞以复杂 方式监测和保持其内恒定的 $\mathrm{H}_{2} \mathrm{O}_{2}$ 水平 ${ }^{[3,4]} \cdot \mathrm{H}_{2} \mathrm{O}_{2}$ 可以 激活许多基因的表达如MAPK激酶等 ${ }^{[76]}$. 同时, $\mathrm{H}_{2} \mathrm{O}_{2}$ 作为植物对病原反应的调节分子, 能选择性诱导一 
些防御基因的表达, 如谷胱甘肽-S-转移酶和谷胱甘 肽氧化酶 ${ }^{[77,78]}$. GSTs作为氧化胁迫过程中的一个保护 酶, 不仅可与GSH结合使膜免遭氧化, 而且还可作为 非酶载体蛋白(配体)参与动物细胞内类固醇、胆红素、 血红素和胆汁盐的转运过程 ${ }^{[79]}$. 尽管植物细胞GST 启动子不含有动物细胞GST启动子中的亲电反应元 件EpRE(electrophile responsive element), 但它却含有 合成章鱼碱合成酶的元件(ocs). 这种含有 $20 \mathrm{bp}$ 的ocs 元件最先是从花梆菜病毒(CaMV)和农杆菌基因启动 子中发现的 ${ }^{[80]}$. 目前为止, 植物细胞中仅有 GSTs 基 因的启动子含有ocs. 此种元件与EpRE有许多相似之 处, 两者都可被氧化胁迫所诱导表达, 可以说, 植物 细胞的GST启动子中ocs元件是氧化胁迫诱导元件. 尽管已经发现 $\mathrm{H}_{2} \mathrm{O}_{2}$ 反应的启动子, 以及植物细胞的 GST启动子中ocs元件是氧化胁迫诱导元件 ${ }^{[40,81]}$, 但 至今仍没有分离和定性 $\mathrm{H}_{2} \mathrm{O}_{2}$ 调控的DNA序列以及与 它们相关的转录因子.

Microarray研究表明, 参与细胞凋亡的基因、信 号转导蛋白(如 $\mathrm{CaM}$ )、蛋白激酶和一些转录因子均可 被 $\mathrm{H}_{2} \mathrm{O}_{2}$ 诱导. $\mathrm{H}_{2} \mathrm{O}_{2}$ 处理后, 在 11000 个基因中 175 个 基因在表达水平上发生了变化 ${ }^{[82]}$. 我们用基因芯片 系统研究拟南芥幼苗中 $\mathrm{H}_{2} \mathrm{O}_{2}$ 引起的基因表达的变化, 发现 $\mathrm{H}_{2} \mathrm{O}_{2}$ 可分别诱导 464 和抑制 221 个基因的表 达 ${ }^{[83]}$. 但是, 对于 $\mathrm{H}_{2} \mathrm{O}_{2}$ 调节的基因转录是否参与细 胞质或细胞核内的氧化变化和转录因子自身修饰, 以及其他修饰反应(如磷酸化) 最终是否由 $\mathrm{H}_{2} \mathrm{O}_{2}$ 启动 目前也知之甚少.

\section{4 结论和展望}

综合最近的研究进展, 植物细胞 $\mathrm{H}_{2} \mathrm{O}_{2}$ 信号转导 可以概括为(图 1): $\mathrm{H}_{2} \mathrm{O}_{2}$ 从质外体或者产生源进入细 胞, 然后扩散进入亚细胞区域. 叶绿体和PMRS系统 可能是 $\mathrm{H}_{2} \mathrm{O}_{2}$ 的主要来源. $\mathrm{H}_{2} \mathrm{O}_{2}$ 可以氧化或者调节信 号转导蛋白, 如蛋白质磷酸化酶(包括质膜组蛋白激 酶和MAPK反应链在内的蛋白质激酶)、转录因子、 以及位于质膜或其它膜上的 $\mathrm{Ca}^{2+}$ 通道. 胞内 $\mathrm{Ca}^{2+}$ 浓度 的升高, 通过钙结合蛋白的作用进一步启动下游的 反应. 蛋白质可逆磷酸化也可启动细胞质和细胞核 的下游信号转导, 通过影响转录因子而影响基因的
表达. 转录因子的氧化可以激活自身或诱导其定向 转运至细胞核内. $\mathrm{H}_{2} \mathrm{O}_{2}$ 的合成可以被Rop信号系统控 制 ${ }^{[44]}$.

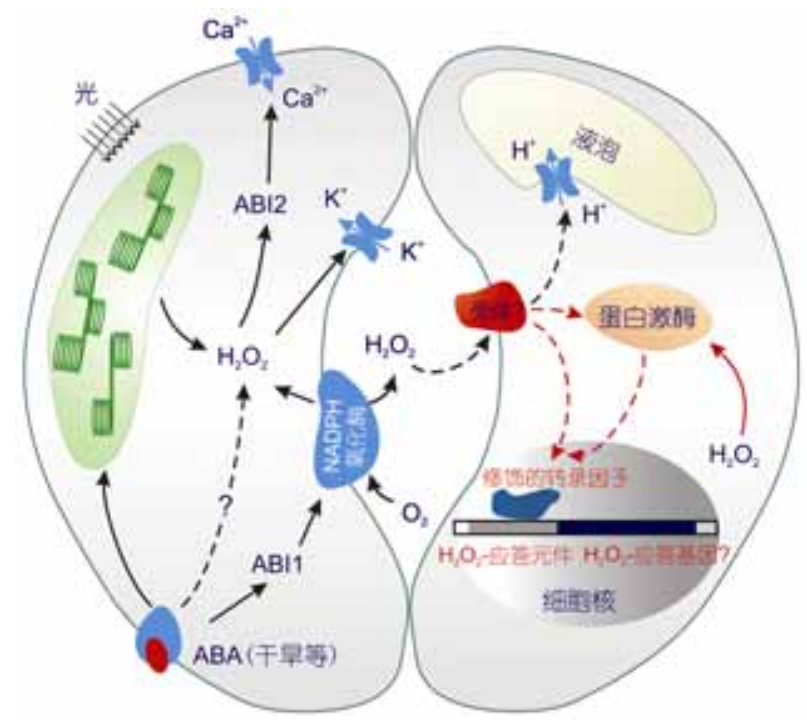

图 1 植物细胞 $\mathrm{H}_{2} \mathrm{O}_{2}$ 信号转导的模式图(以保卫细胞为例) 外界刺激可以诱导 $\mathrm{H}_{2} \mathrm{O}_{2}$ 产生生苜, 多8 , 然后扩散进入亚细胞区域. $\mathrm{H}_{2} \mathrm{O}_{2}$ 可 以氧化或者调节信号转导蛋白, 如蛋白质磷酸化酶(例如, ABI1 和 $\mathrm{ABI} 2)^{[59]}$ 、蛋白激酶 ${ }^{[69]}$ 、转录因子 ${ }^{[40]}$, 以及位于质膜或其它膜上的 $\mathrm{K}^{-}$ 和 $\mathrm{Ca}^{2+}$ 通道 ${ }^{[38,400}$. 胞内 $\mathrm{Ca}^{2+}$ 浓度的升高, 通过钙结合蛋白的作用进一 步启动下游的反应. 蛋白质可逆磷酸化也可启动细胞质和细胞核的下 游信号转导, 通过影响转录因子而影响基因的表达. 转录因子的氧化 可以激活自身或诱导其定向转运至细胞核内. 红色虚线部分示目前仍 然未知的信号转导途径, 也是未来研究的重点

从进化的角度, 无疑 $\mathrm{H}_{2} \mathrm{O}_{2}$ 是一个古老的信号, 尤其是氧化还原是生命的基本过程, 在许多生理生 化过程和基因转录的许多方面有着非常重要的作用. 但是对于 $\mathrm{H}_{2} \mathrm{O}_{2}$ 作为信号分子的其信号转导途径的详 细过程了解仍然是相对年轻的课题, 许多基本的问 题仍然不清楚. 随着对 $\mathrm{H}_{2} \mathrm{O}_{2}$ 相关研究的不断深入, 使用转基因植物和 $\mathrm{H}_{2} \mathrm{O}_{2}$ 信号转导的突变体来阐明特 异细胞和不同刺激情形下 $\mathrm{H}_{2} \mathrm{O}_{2}$ 的生物学功能; 还可 能通过分析一些基因组成的变化，在植物中发现 $\mathrm{H}_{2} \mathrm{O}_{2}$ 作用的专门元件如 $\mathrm{H}_{2} \mathrm{O}_{2}$ 的结合蛋白, $\mathrm{H}_{2} \mathrm{O}_{2}$ 的受 体等, 从而更加清楚地了解 $\mathrm{H}_{2} \mathrm{O}_{2}$ 在植物细胞内部尤 其是气孔保卫细胞内的信号转导及作用.

尽管有较多的实验表明, ABA 可诱导保卫细胞 
中 $\mathrm{H}_{2} \mathrm{O}_{2}$ 的产生和积累, 而 $\mathrm{H}_{2} \mathrm{O}_{2}$ 又作为胞内信号成分 构成了 ABA 信号转导体系的一个重要环节, 从而推 测叶绿体的光电子传递系统、线粒体的电子传递体 系和质膜上 $\mathrm{NADPH}$ 氧化酶等与 $\mathrm{ABA}$ 诱导的 $\mathrm{H}_{2} \mathrm{O}_{2}$ 产生有关. 但是, 没有人在亚细胞水平上对 $\mathrm{ABA}$ 诱 导的 $\mathrm{H}_{2} \mathrm{O}_{2}$ 的产生进行定位, 因而对保卫细胞内 ABA 诱导的 $\mathrm{H}_{2} \mathrm{O}_{2}$ 的产生进行亚细胞定位就显得格外重要.

$\mathrm{H}_{2} \mathrm{O}_{2}$ 可以调节气孔的运动. 近 20 年来, 由于基 因组学的发展和进步, 在植物生物学的其他领域如 发育、矿质营养和抗盐等方面, 使用分子遗传学技术, 尤其是基于拟南芥模式系统的研究, 都已经发生了 革命性的变化. 然而, 在植物对保卫细胞信号转导的 研究中, 利用拟南芥模式体系几乎没有成功的先例, 因为至今没有一个非常直观的观察方法来监测气孔 的反应, 无法建立起有效的分子遗传学的䇻选体系. 目前, 研究保卫细胞内基因调控模式遇到的最大困 难是确定这些基因是否在保卫细胞信号转导过程中 起作用, 至今尚末发现 $\mathrm{H}_{2} \mathrm{O}_{2}$ 特异的感受子和受体, 也就是说我们仍不知道细胞如何感受 $\mathrm{H}_{2} \mathrm{O}_{2}$, 以及许 多引起 ABA 反应的基因在保卫细胞反应过程中的功 能, 同时, 我们也不知道在细胞感受 $\mathrm{H}_{2} \mathrm{O}_{2}$ 信号转导 过程中哪种细胞过程是最主要的或是限速步骤, 更 不知道何种基因对 $\mathrm{H}_{2} \mathrm{O}_{2}$ 是特异和必需的, 以及该基 因是否在特异组织中表达, 所有这些问题的解决, 都 要依赖于保卫细胞内功能性的遗传学和基因组学分 析.

致谢 感谢河南大学生命科学学院王鹏程、江静、苗 雨晨、郝福顺、吕东、王棚涛、萄红帮助整理部分内 容.

\section{参考文献}

1 Jamieson D J, Storz G. In: Scandalios J G, ed. Oxidative Stress and the Molecular Biology of Antioxidant Defenses. New York: Cold Spring Harbor Laboratory Press, 1997. 91 115

2 Foyer C H, Noctor G. Redox homeostasis and antioxidant signaling: A metabolic interface between stress perception and physiological responses. Plant Cell, 2005, 17: 1866 1875[DOI]

3 Neill S J, Desikan R, Hancock J T. Hydrogen peroxide signalling. Curr Opin Plant Biol, 2002, 5: 388 395[DOI]

4 Apel K, Hirt H. Reactive oxygen species: Metabolism, oxidative stress, and signal transduction. Annu Rev Plant Bio, 2004, 55,
373 399[DOI]

5 MeKay C P, Hartman H. Hydrogen peroxide and the evolution of oxygenic photosynthesis. Orig Life Envol Biosph, 1991, 21: 157 163[DOI]

6 Karpinski S, Reynolds H, Karpinska B, Wingsle G, Creissen G, Mullineaux P. Systemic signaling and acclimation in response to excess excitation energy in Arabidopsis. Science, 1999, 284: $654 \sim 657[\mathrm{DOI}]$

7 Jiang M, Zhang J. Effect of abscisic acid on active oxygen species, antioxidant defencesystem and oxidative damage in leaves of maize seedlings. Plant Cell Physiol, 2001, 42: 1265 1273[DOI]

8 Allan A C, Fluhr R. Two district sources of elicited reactive oxygen species in tobacco epidermal cells. Plant Cell, 1997, 9: 1559 1572[DOI]

9 Alvarez M E, Lamb C. Oxidative burst-mediated defense responses in plant disease resistance. In: Scandalios J G, ed. Oxidative Stress and the Molecular Biology of Antioxidant Defenses. New York: Cold Spring Harbor Laboratory Press, 1997. 815 839

10 Sone J R. An assessment of proposed mechanism for sensing hydrogen peroxide in mammalian systems, Arch Biochem Biophys, 2004, 422: 119 124[DOI]

11 Rubinstein B, Luster D G. Plasma membrane redox activity: Components and role in plant processes. Annu Rev Plant Physiol Plant Mol Biol, 1993, 44: 131 155[DOI]

12 Torres M A, Dangl J L, Jones J D G. Arabidopsis gp9 $1^{\text {phox }}$ homologues AtrbohD and AtrbohF are required for accumulation of reactive oxygen intermediates in the plant defense response. Proc Natl Acad Sci USA, 2002, 99: 517 522[DOI]

13 Kwak J M, Mori I C, Torres M A, et al. NADPH oxidase AtrbohD and AtrbohF genes function in ROS-dependent ABA signaling in Arabidopsis. EMBO J, 2003, 22: 2623 2633[DOI]

14 Yoshioka $\mathrm{H}$, Numata $\mathrm{N}$, Doke $\mathrm{N}$, et al. Nicotiana benthamiana gp91phox homologs NbrbohA and NbrbohB participate in $\mathrm{H}_{2} \mathrm{O}_{2}$ accumulation and resistance to Phytophthora infestans. Plant Cell, 2003, 15: 706 718[DOI]

15 Lamb C, Dixon R A. The oxidative burst in plant disease resistance. Annu Rev Plant Physiol Plant Mol Biol, 1997, 48: 251 275[DOI]

16 Sagi M, Fluhr R. Superoxide production by plant homologues of the gp9 $1^{\text {phox }}$ NADPH oxidase. Modulation of activity by calcium and by tobacco mosaic virus infection. Plant Physiol, 2001, 126: 1281 1290[DOI]

17 苗雨晨, 宋纯鹏, 董发才, 王学臣. ABA 诱导虽豆气孔保卫细胞 $\mathrm{H}_{2} \mathrm{O}_{2}$ 的产生. 植物生理学报, 2000, 26 (1): 53 58 
18 Zhang X, Zhang L, Song C P, et al. Hydrogen peroxide is involved in abscisic acid-induced stomatal closure in Vicia faba. Plant Physiol, 2001, 126: 1438 1448[DOI]

19 Peng M, Kuc J. Peroxidase-generated hydrogen peroxide as a source of antifungal activity in vitro and on tobacco leaf disks Phytopathol, 1992, 82: 696 699

20 Bolwell G P, Butt V S, Zimmerlin A, et al. The origin of the oxidative burst in plants. Free Radical Res, 1995, 23: 517 532

21 Hernández J A, Jiménez A, Sevilla F, et al. Tolerance of pea (Pisum sativum L.) to long-term salt stress is associated with induction of antioxidant defences. Plant Cell Environ, 2000, 23: $853 \sim 862[\mathrm{DOI}]$

22 Grant J, Yun B W, Loake G J. Oxidative burst and cognate redox signalling reported by luciferase imaging: identification of a signal network that functions independently of ethylene, SA and Me-JA but is dependent on MAPKK activity. Plant J, 2000, 24: 569 582[DOI]

23 Yang T, Poovaiah B W. Hydrogen peroxide homeostasis: Activation of plant catalase by calcium/calmodulin. Proc Natl Acad Sci USA, 2002, 99: 4097 4102[DOI]

24 Vandenabeele S, Vanderauwera S, Vuylsteke M, et al. Catalase deficiency drastically affects gene expression induced by high light in Arabidopsis thaliana. Plant J, 2004, 39: 45 58[DOI]

25 Dutilleul C, Garmier M, Noctor G, et al. Leaf mitochondria modulate whole cell redox homeostasis, set antioxidant capacity, and determine stress resistance through altered signaling and diurnal regulation. Plant Cell, 2003, 15: 1212 1226[DOI]

26 Devletova S, Rizhsky L, Mittler R, et al. Cytosiolic ascorbate peroxides 1 is a central component of the reactive oxygen gene network of Arabidopsis. Plant Cell, 2005, 17: 268 281[DOI]

27 Chen Z, Gallie D R. The ascorbic acid redox state controls guard cell signaling and stomatal movement. Plant Cell, 2004, 16: 1143 1162[DOI]

28 Milla M A, Maurer A, Gustafson J P, et al. Glutathione peroxidase genes in Arabidopsis are ubiquitous and regulated by abiotic stresses through diverse signaling pathways. Plant J, 2003, 36 : 602 615[DOI]

29 Delaunay A, Pflieger D, Toledano M B, et al. A thiol peroxidase is an $\mathrm{H}_{2} \mathrm{O}_{2}$ receptor and redox-transducer in gene activation. Cell, 2002, 111: 471 481[DOI]

30 Singh K K. The Saccharomyces cerevisiae Sln1p-Ssk1p twocomponent system mediates response to oxidative stress and in an oxidant-specific fashion. Free Radic Biol Med, 2000, 29: 1043 1050[DOI]

31 Song $\mathrm{C} \mathrm{P}, \mathrm{Du} \mathrm{Z}, \mathrm{Zhu} \mathrm{Z}$, et al. $\mathrm{NaHSO}_{3}$ inhibits photorespiration by decreasing the production of superoxde anion. In: Asada K,
Yashikawa T, eds. Frontiers of Reactive Oxygen Species in Biology and Medicine. Amsterdam: Elsevier Science BV, 1994. 35 36

32 Noctor G, Gomez L, Foyer $\mathrm{C} \mathrm{H}$, et al. Interactions between biosynthesis, compartmentation and transport in the control of glutathione homeostasis and signaling. J Exp Bot, 2002, 53: 1283 1304[DOI]

33 Baker C J, O'Neill N R, Orlandi E W, et al. Early responses during plant-bacteria interactions in tobacco cell suspensions. Phytopathol, 1991, 81: 1504 1507

34 Levine A, Pennell R I, Lamb C, et al. Calcium-mediated apoptosis in a plant hypersensitive disease resistance response. Curr Biol, 1996, 6: 427 437[DOI]

35 Chen Z X, Silva H, Klessig D F. Active oxygen species in the induction of plant systemic acquired resistance by salicylic acid. Science, 1993, 262: 1883 1886

36 Shirasu K, Nakajima H, Lamb C, et al. Salicylic acid potentiates an agonist-dependent gain control that amplifies pathogen signals in the activation of defense mechanisms. Plant Cell, 1997, 9: 261 270[DOI]

37 Dong F C, Wang P T, Song C P, et al. The role of hydrogen peroxide in Salicylic acid-induced stomatal closure in Vicia faba guard cells. Acta Phytophysicol Sin, 2001, 27: 296 302

38 Pei Z M, Murata Y, Schroeder J L, et al. Calcium channels activated by hydrogen peroxide mediate abscisic acid signaling in guard cells. Nature, 2000, 406: 731 734[DOI]

39 Morris P C. MAP kinase signal transduction pathways in plants. New Phytol, 2001, 151: 67 89[DOI]

40 Kovtun Y, Chiu W L, Sheen J, et al. Functional analysis of oxidative stress-activated mitogen-activated protein kinase cascade in plants. Proc Natl Acad Sci USA, 2000, 97: 2940 2945[DOI]

41 Moon H, Lee B, Parasad T, et al. NDP kinase 2 interacts with two oxidative stress-activated MAPKs to regulate redox state and enhances multiple stress tolerance in transgenic plants. Proc Natl Acad Sci USA, 2003, 100: 358 363[DOI]

42 江 静, 安国勇, 王鹏程, 等. MAP 激酶调节蚕豆保卫细胞中 $\mathrm{ABA}$ 诱导的 $\mathrm{H}_{2} \mathrm{O}_{2}$ 产生, 科学通报, 2003, 48(12): 1256 1263

43 Yang Z. Small GTPases: Versatile signalling switches in plants. Plant Cell, 2002, 14: S375 S388

44 Baxter-Burrell A, Yang Z, Bailey-Serres J, et al. RopGAP4dependent Rop GTPase rheostat control of Arabidopsis oxygen deprivation tolerance. Science, 2002, 296: 2026 2028[DOI]

45 Dangl, J. Plants just say NO to pathogens. Nature, 1998, 394: $525 \sim 526[\mathrm{DOI}]$

46 Zhao Z, Chen G, Zhang C. Interaction between reactive oxygen species and nitric oxide in drought-induced abscisic acid synthesis in root tips of wheat seedlings. Aust J Plant Physiol, 2001, 28: 
$1055 \sim 1061$

47 Garcia-Mata C, Lamattina L. Nitric oxide induces stomatal closure and enhances the adaptive plant responses against drought stress. Plant Physiol, 2001, 126: 1196 1204[DOI]

48 Beligni M V, Lamattina L. Nitric oxide interferes with plant photo-oxidative stress by detoxifying reactive oxygen species. Plant Cell Environ, 2002, 25: 737 748[DOI]

49 Desikan R, Griffiths R, Neill S J, et al. A new role for an old enzyme: Nitrate reductase-mediated nitric oxide generation is required for abscisic acid-induced stomatal closure in Arabidopsis thaliana. Proc Natl Acad Sci USA, 2002, 25: 16314 16318[DOI]

50 Garcia-Mata C, Gay R, Blatt M R, et al. Nitric oxide regulates $\mathrm{K}^{+}$ and $\mathrm{Cl}^{-}$channels in guard cells through a subset of abscisic acid-evoked signaling pathways. Proc Natl Acad Sci USA, 2003, 100: 11116 11121[DOI]

51 Neill S J, Desikan R, Hancock J T, et al. Nitric oxide is a novel component of abscisic acid signaling in stomatal guard cells. Plant Physiol, 2002, 128: 13 16[DOI]

52 Neill SJ, Desikan R, Hancock J T. Nitric oxide as a mediator of ABA signalling in stomatal guard cells. Bulg J Plant Physiol, 2003 , Special Issue: $124 \sim 132$

53 Lv D, Zhang X, Song C P, et al. NO may function in the downstream of $\mathrm{H}_{2} \mathrm{O}_{2}$ in ABA-induced stomatal closure in Vicia faba L. J Plant Physiol Mol Biol 2005, 31: 62 37

54 Lamattina L, Garcia-Mata C, Pagnussat G, et al. Nitric oxide: The versatility of an extensive signal molecule. Annu Rev Plant Biol, 2003, 54: 109 136[DOI]

55 Desikan R, Cheung M K, Neill S J, et al. ABA, hydrogen peroxide and nitric oxide signalling in stomatal guard cells. J Exp Bot, 2004, 55: 205 212[DOI]

56 Neill S J, Desikan R, Hzncock J T, et al. Hydrogen peroxide and nitric oxide as signalling molecules in plants. J Exp Bot, 2002, 372: 1237 1247[DOI]

57 Delledonne M, Zeier J, Lamb C, et al. Signal interactions between nitric oxide and reactive oxygen intermediates in the plant hypersensitive disease resistance response. Proc Natl Acad Sci USA 2001, 98: 13454 13459[DOI]

58 Lum H K, Butt Y K C, Lo S C L. Hydrogen peroxide induces a rapid production of nitric oxide in mung bean (Phaseolus aureus). Nitric Oxide, 2002, 2: 205 213[DOI]

59 Murata Y, Pei Z M, Schroeder J I, et al. Abscisic acid activation of plasma membrane $\mathrm{Ca}^{2+}$ channels in guard cells requires cytosolic $\mathrm{NAD}(\mathrm{P}) \mathrm{H}$ and is differentially disrupted upstream and downstream of reactive oxygen species production in abi1-1 and abi2-1 protein phosphatase 2C mutants. Plant Cell, 2001, 13: $2513 \sim 2523$ [DOI]
60 Miura Y, Yoshioka H, Doke N. An autophotographic determination of the active oxygen generation in potato tuber discs during hypersensitive responses to fungal infection or elicitor. Plant Sci, 1995, 105: 45 52[DOI]

61 Tavernier E, Wendehenne D, Pugin A, et al. Involvement of free calcium in action of cryptogein, a proteinaceous elicitor of hypersensitive reaction in tobacco cells. Plant Physiol, 1995, 109: $1025 \sim 1031$

$62 \mathrm{Xu} \mathrm{H}$, Heath M C. Role of calcium in signal transduction during the hypersensitive response caused by basidiospore-derived infection of the cowpea rust fungus. Plant Cell, 1998, 10: 585 597[DOI]

63 McAinsh M R, Clayton H, Hetherington A M, et al. Changes in stomatal behavior and cytosolic free calcium in response to oxidative stress. Plant Physiol, 1996, 111: 1031 1042

64 An G, Song C P, Zhang X, et al. Effect of peroxide generation on stomatas movement and $\mathrm{K}^{+}$channel on plasma membrane in Vicia faba guard cell. Acta Phytophysiol Sin, 2000, 26 (5): 458 464

65 Zhang X, Miao Y, Song C P, et al. $\mathrm{K}^{+}$channels inhibited by hydrogen peroxide mediate abacisic acid signaling in Vicia faba guard cells. Cell Res, 2001, 11: 195 202

66 Zhang X, Dong F C, Gao J, Song C P. Hydrogen peroxide-induced changes in intracellular $\mathrm{pH}$ of guard cells precede stomatal closure. Cell Res, 2001: 11: 37 43

67 Schroeder J I, Kwak J M, Allen G J. Guard cell abscisic acid signaling and engineering drought hardiness in plants. Nature, 2001, 410: 327 330.[DOI]

68 Park K Y, Jung J Y, Lee Y, et al. A role for phosphatidylinositol 3-phosphate in abscisic acid-induced reactive oxygen species generation in guard cells. Plant Physiol, 2003, 132: 92 98[DOI]

69 Mustilli A C, Merlot S, Giraudat J, et al. Arabidopsis OST1 protein kinase mediates the regulation of stomatal aperture by abscisic acid and acts upstream of reactive oxygen species production. Plant Cell, 2002, 14: 3089 3099[DOI]

70 Bestwick C S, Brown I R, Mansfield J W, et al. Localization of hydrogen peroxide accumulation during the hypersensitive reaction of Lettuce cells to Pseudomonas springae pv phaseolicola. Plant Cell, 1997, 9: 209 221[DOI]

71 Tiwari B S, Belenghi B, Levine A. Oxidative stress increased respiration and generation of reactive oxygen species, resulting in ATP depletion, opening of mitochondrial permeability transition, and programmed cell death. Plant Physiol, 2002, 128 : 1271 1281[DOI]

72 Ren D, Yang H, Zhang S. Cell death mediated by MAPK is associated with hydrogen peroxide production in Arabidopsis. J Biol Chem, 2002, 277: 559 565[DOI] 
73 Bauerle P A, Baltimore D. NF-кB: Ten years after. Cell, 1996, 87: 13 20[DOI]

74 Ryals J, Weymann K, Ellis D, et al. The Arabidopsis NIM1 protein shows homology to the mammalian transcription factor inhibitor IкB. Plant Cell, 1997, 9: 425 439[DOI]

75 Xanthoudakis S, Curran T. Identification and characterization of Ref-1, a nuclear protein that facilitates AP-1 DNA-binding activity. EMBO J, 1992, 11: 653 665

76 Desikan R, Clarke A, Neill S J, et al. $\mathrm{H}_{2} \mathrm{O}_{2}$ activates a MAP kinase-like enzyme in Arabidopsis thaliana suspension cultures. J Exp Bot, 1999, 50: 1863 1866[DOI]

77 Levine A, Tenhaken $\mathrm{R}$, Lamb $\mathrm{C}$, et al. $\mathrm{H}_{2} \mathrm{O}_{2}$ from the oxidative burst orchestrates the plant hypersensitive disease resistance response. Cell, 1994, 79: 583 593[DOI]

78 Marrs K A. The functions and regulation of glutathione Stransferases in plants. Annu Rev Plant Physiol Plant Mol Biol, 1996, 47: 127 158[DOI]

79 Listowski I, Abramovitz M, Niitsu Y, et al. Intracellular binding and transport of hormones and xenobiotics by glutathione S-transferases. Drug Metab Rev, 1988, 19: 305 318

80 Zhang B, Singh K B. Ocs element promoter sequences are activated by auxin and salicylic acid in Arabidopsis. Proc Natl Acad Sci USA, 1994, 91: 2507 2511

81 Ulmasov T, Ohmiya A, Guilfoyle T, et al. The soybean GH2/4 gene that encodes a glutathione S-transferases has a promoter that is activated by a wide range of chemical agents. Plant Physiol, 1995, 108: 919 927[DOI]

82 Desikan R, Mackerness S A H, Neill S J, et al. Regulation of the Arabidopsis transcriptome by oxidative stress. Plant Physiol, 2001, 127: 159 172[DOI]

83 Wang P C, Du Y Y, An G Y, Zhou Y, Song C P. Analysis of global expression profiles of Arabidopsis genes under abscisic acid and $\mathrm{H}_{2} \mathrm{O}_{2}$ applications. Journal of Integrative Plant Biology, 2006, 48: $62 \sim 74$ 